\title{
Introducing the Urban Climate Model PALM System 6.0
}

\author{
Mohamed Hefny Salim* \\ Faculty of Energy Engineering \\ Aswan University \\ Aswan, Egypt \\ mohamed.hefny@aswu.edu.eg
}

\author{
Christoph Schneider \\ Geography Department \\ Humboldt-Universität zu Berlin \\ Berlin, Germany \\ christoph.schneider@geo.hu-berlin.de
}

\author{
Sebastian Schubert \\ Geography Department \\ Humboldt-Universität zu Berlin \\ Berlin, Germany \\ sebastian.schubert@geo.hu-berlin.de
}

\author{
Björn Maronga \\ Institute of Meteorology and Climatology \\ Leibniz University Hannover \\ Hannover, Germany \\ maronga@muk.uni-hannover.de
}

\author{
Mohamed Fathy Cidek \\ Faculty of Energy Engineering \\ Aswan University \\ Aswan, Egypt \\ mohamed.fathy@aswu.edu.eg
}

\begin{abstract}
This contribution describes the urban climate model system PALM 6.0 for applications in urban areas (Palm4U). PALM4U contains several components to enable the model to simulate the atmospheric processes in urban areas on a building resolving scale. These components include the following modules: a land- and urban surface model, a chemistry model, a wind turbine model, a plant canopy model, human biometeorology model, an indoor and building energy model, and a radiation model. A study case representing a realistic urban domain is designed and simulated to show the model capabilities. The chosen domain is located in the Charlottenburg neighbourhood in Berlin, Germany. In this study we show the flow (wind field) as well as the thermal (radiation and temperature) properties as an example of a typical model application. The study emphasises on the potential of using such an urban climate model as a design tool for urban planing strategies, planning of renewable energy systems, and the investigation of implications of climate change.
\end{abstract}

Index Terms-Urban climate, flow modelling, simulation, urban planning, renewable energy

\section{INTRODUCTION}

\section{A. Urban climate modelling}

In order to study the interaction between urban environments and the atmosphere we need reliable tools, based on physical and/or modelling approaches. Urban climate models (UCMs) are useful tools to accomplish such studies accurately and to serve as modern tools for urban planning. These models are broadly classified into two main model types, namely urban canopy-layer models and urban boundary-layer models [1]. Models of the first type are used to investigate microscale climate variations occurring below canopy-height while models of the second type are utilised to examine the mesoscale climate variations occurring above canopy-level.

The recent development of models based on the first type has been mainly driven by the advances in computer capacities

Funding agency: the German Federal Ministry of Education and Research (BMBF), grant 01LP1601.

*Also at Humboldt-Universität zu Berlin, Berlin, Germany. and numerical algorithms [2]. Also, over the last decades, there are advances in the parameterization of micrometeorological processes and data availability for urban area, which imposes an increasing interest on the first category of UCMs. UCMs are now being widely used to study the implications and possible adaptation measures of climate change decisions for urban areas.

\section{B. The PALM model system 6.0}

The PALM model system 6.0 (PALM4U) is developed to be a modern and highly efficient UCM allowing for simulations over a neighbourhood- and at full city-scale with buildingresolving spatial resolution [3]. The model system is developed within the framework of the joint project MOdel-baSed city planning and Application In climate change (MOSAIK). MOASIK is funded within the programme Urban Climate Under Change ([UC]2) by the German Federal Ministry of Education and Research (BMBF). The model is basically based on version 4.0 of the model PALM, the well-established largeeddy simulation (LES) model. [4]. The model is equipped with all the components required for simulating urban areas. It includes perfect scalability on massively parallel computer architectures. Its performance is evaluated against field measurements and previous simulations [6]. The model is briefly described in this paper, however for detailed description readers are advised to refer to the detailed description of the model reported in Maronga et al. [1].

\section{Objectives}

The objective of this paper is to introduce the PALM4U as a useful tool for urban climate modelling. It highlights the importance of using a model to examine the planning strategies and to enable decision support in urban planning. 


\section{MODEL DESCRIPTION}

\section{A. PALM model core}

PALM4U solves the three-dimensional, non-hydrostatic, filtered, incompressible Navier-Stokes equations of wind ( $u, v$, and $w$ ) and scalar variables (sub-grid-scale (SGS) turbulent kinetic energy, potential temperature, and specific humidity) on a staggered Cartesian grid. To neglect density variations except for the buoyancy term, the Boussinesq-approximation is applied to the filtered Navier-Stokes equations. The model has two modes for turbulence parameterizations: the LES and the Reynolds-averaged Navier-Stokes (RANS) mode. The SGS turbulence parameterization depends on the mode of simulation being LES or RANS. As turbulence closures the model offers the 1.5-order closure and a dynamic SGS closure in the LES mode. For RANS mode the model offers twoequation turbulence model closures, $k-l$ and $k-\epsilon$ where $k$ is the turbulent kinetic energy, $l$ is a mixing length and $\epsilon$ is the dissipation rate of SGS turbulent kinetic energy.

\section{B. PALM model components}

PALM4U is equipped with many components (modules) needed to perform simulations related to the atmospheric processes in urban areas. It has the following components:

- Radiation module. This module provides the solar radiation (longwave and shortwave fluxes) throughout the domain. It is important for other components of the model as it serves as the input to these models. It incorporates a simple radiation model, i.e. the clear-sky model, and a more sophisticated model, i.e. the Rapid Radiative Transfer Model for Global models (RRTMG).

- Wind turbine model. It enables PALM4U simulating wind turbines located in the domain. It provides a detailed view of the wake effect of the wind turbine, especially how the atmospheric boundary layer affects the wake and vice versa.

- Land surface module. This module performs the energy balance for all natural surfaces such as water and vegetation surfaces.

- Urban surface module. The module computes the energy balance for all urban surfaces, more specifically all artificial surfaces such as building facade, roofs, pavements, etc.

- Chemistry module. These components allow for air quality related studies by simulating the emissions, the chemical transformation and dispersion, as well as the deposition of different chemical components in the urban domain, i.e. NOx, Particulate Matter (PM) and COx.

- Indoor and building energy demand model. This component determines the energy demand for heating and cooling and the indoor thermal environment of buildings by performing the energy balance of the interior of buildings.

- Multi-agent system. This model simulates individual pedestrians moving in a crowd embedded in the atmospheric model in order to evaluate the quality of life for individuals and the environmental effects on large groups of people. Also, it can simulate evacuation scenarios in case of escape situations.

- Human biometeorology module. It deals with all processes related to calculating the outdoor human comfort, and it calculates the related thermal comfort indices. Plant canopy module. In this module all the processes related to large vegetation,such as trees and shrubs are considered to account for the vertically extended drag, release of heat, plant evapotranspiration and leaf-airinteractions.

\section{PALM model availability}

PALM4U can be downloaded from the official model web-page: http://palm-model.org. It is distributed under the GNU General Public License v3 (http://www.gnu.org/copyleft/gpl.html). Code management and versioning is done via an Apache Subversion (http://subversion.apache.org) (svn) server. Furthermore, the model is supported by a web-based project management and bug-tracking system using the software Trac (http://trac.edgewall.org) for monitoring recent code changes and reporting bugs through a ticketing system directly to the developers of the code.

\section{Simulation EXAMPLE}

\section{A. Study case}

The study case chosen here to show the capabilities of PALM4U is a domain extending $1 \times 1 \mathrm{~km}^{2}$ in a neighbourhood around the town square Ernst-Reuter-Platz located in Charlottenburg in Berlin (Germany). This is a realistic urban configuration, as shown in Fig. 1. The domain comprises all typical features of urban complexity required for this purpose. For instance, it comprises different building heights, street configurations, trees, and open spaces. The available information of real building configuration and the $3 \mathrm{D}$ structure of vegetation (street trees and parks) are utilised to integrate the buildings and vegetation into the computational domain. Also, the real orography heights and the surface cover characteristics of this domain are included. The data are provided by the German Aerospace Center (DLR).

\section{B. Results and discussion}

The full 3-D simulation is carried out for a typical summer day (30th June) and the simulation time is 2 days under clearsky conditions (no cloud formation). The surface temperature is properly initialised before the 3-D simulation by a precursor simulation for one day using the PALM spin-up mechanism to save computational load. The simulation was running on 1024 computer cores 5.55 wall-clock hours (5684.92 in total). The flow field in the domain is depicted in Fig. 2-4 showing the $u, v$, and $w$ wind components. The effect of buildings as well as the different land cover becomes clear from the figures. All typical flow characteristics are captured by the model. For instance, the effects of buildings on the flow field are seen in several flow features such as wind speed acceleration in open 


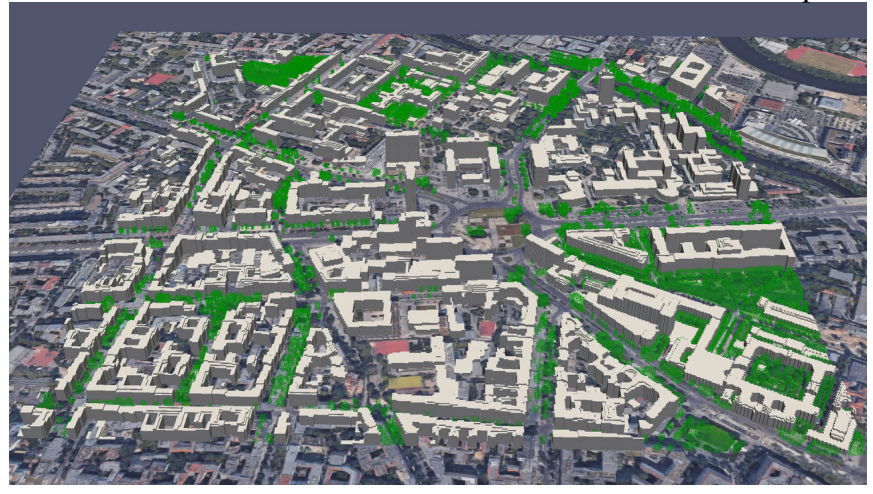

Fig. 1. Aerial view of the 3-D structure of buildings and vegetation (trees and parks, shown as green points). The domain is centred around Ernst-ReuterPlatz in Charlottenburg in Berlin (N52 $2^{\circ} 30.8^{\prime} \mathrm{E} 48^{\circ} 19.31^{\prime}$ ). The copyright for the underlying satellite image is held by GeoBasis-DE/BKG (@2009), Google.

areas, deceleration within dense building configurations, and up-drafts and down-drafts around buildings.

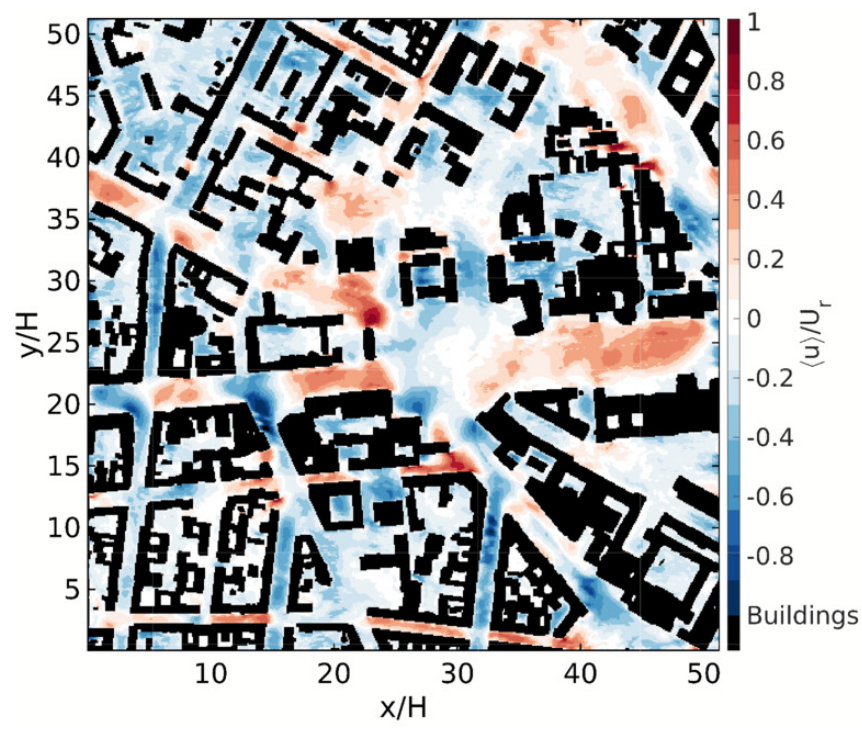

Fig. 2. Wind component $u$ in the simulated area (Ernst-Reuter-Platz in Charlottenburg, Berlin) during a summer day.

The air temperature near the surface is affected by the radiation interactions within the urban realm as well as the flow field. Figure 5 shows the near surface air temperature in the domain at night time. It can clear be shown from the figure that air temperature is very heterogeneous due to the complex flow and radiation processes resulting from the spatial complexity of the urban domain and the vegetation elements.

One of the important and challenging characteristics of the urban area is the heterogeneity of the surfaces. Each surface has its own surface characteristics and is exposed to different radiation flux. Consequently, the surface temperature is different for each surface. PALM4U can calculate the surface temperature of each surface.

Figure 6 shows the surface temperature of a focus domain at midday at 13:00. It can be seen from the figure that the surface

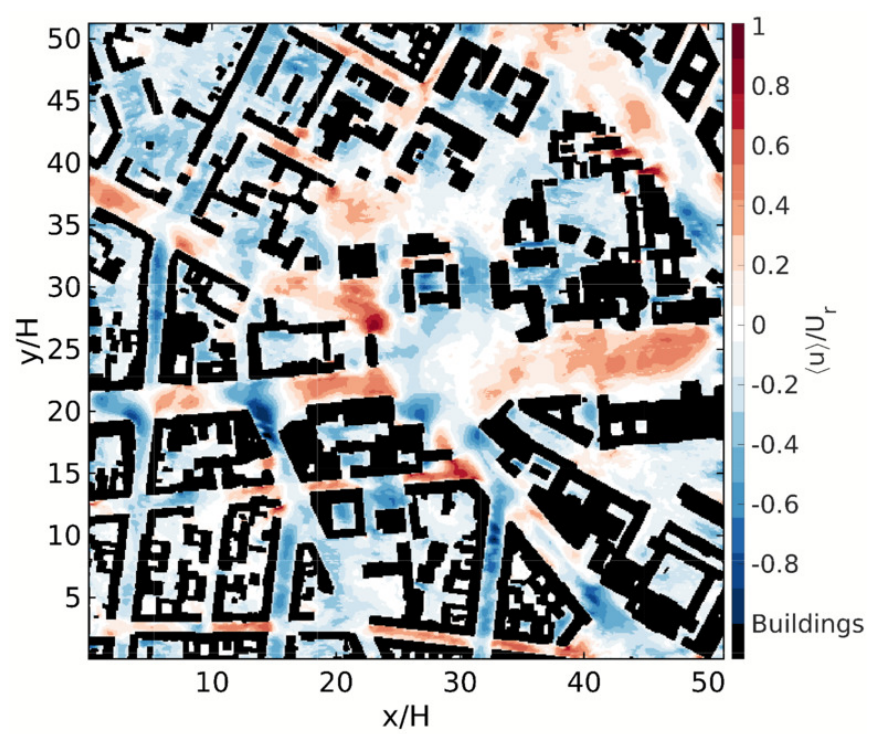

Fig. 3. Wind component $v$ in the simulated area (Ernst-Reuter-Platz in Charlottenburg, Berlin) during a summer day.

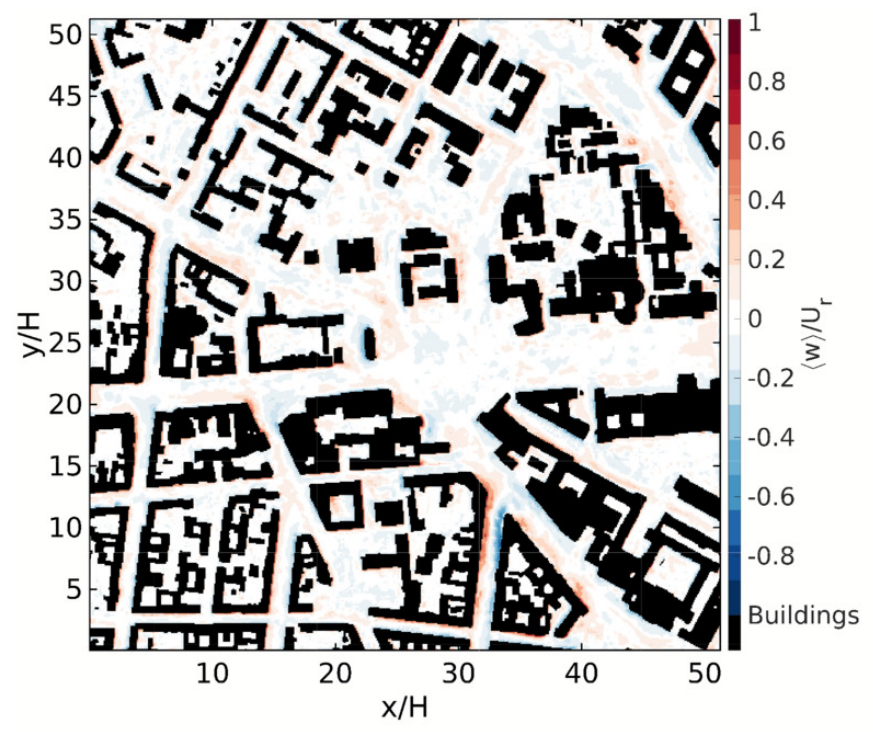

Fig. 4. Wind component $w$ in the simulated area (Ernst-Reuter-Platz in Charlottenburg, Berlin) during a summer day.

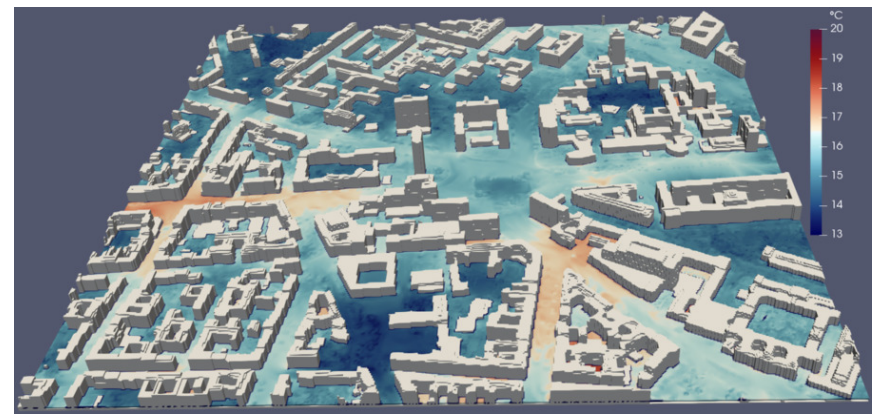

Fig. 5. Near surface air temperature in the simulated domain (Ernst-ReuterPlatz in Charlottenburg, Berlin) during a summer night. 
temperature depends on the type, location, and orientation of the surface.

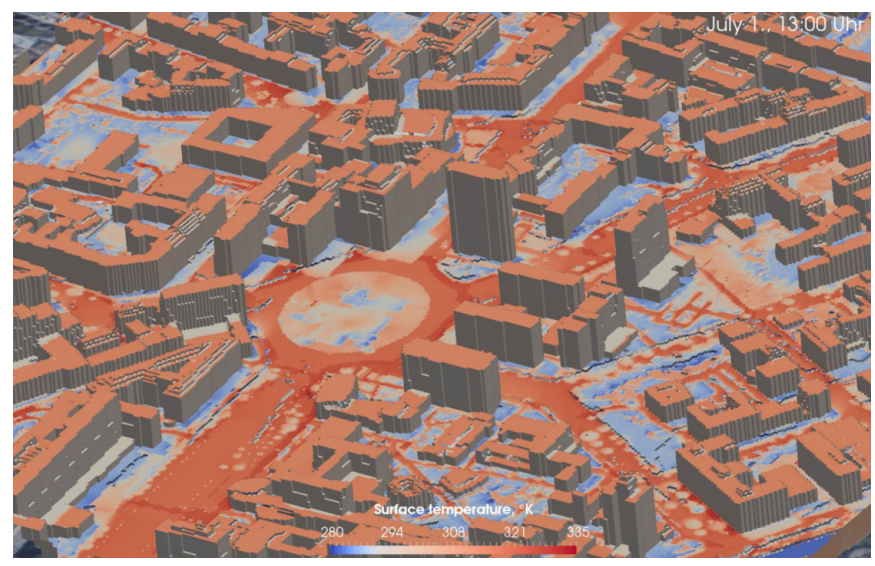

Fig. 6. Surface temperature of a focus domain in the simulated area (ErnstReuter-Platz in Charlottenburg, Berlin) during a summer day at 13:00.

The radiation model integrated in PALM4U is capable to accurately predict shortwave and longwave radiation fluxes received by each surface. It accounts for the obstacles and the vegetation (trees) as well as the orientation of the surfaces.

Figure 7 shows the shortwave radiation flux received by each surface in the morning. Building shadows and tree shadows can be seen in the figure. Both have a major impact on the radiation budget of the surfaces, and hence the surface temperature.

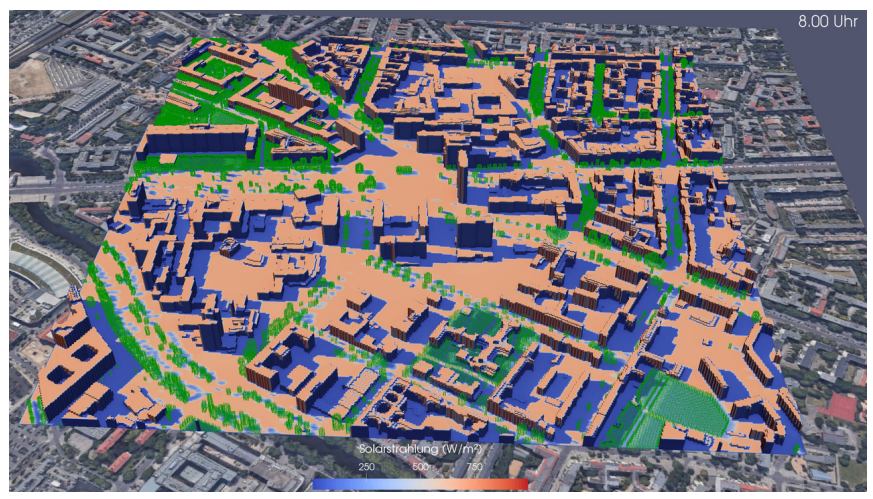

Fig. 7. Solar radiation (shortwave) flux received by each surface in a focus domain in the simulated area (Ernst-Reuter-Platz in Charlottenburg, Berlin) during a summer day at 08:00.

Since urban surfaces and vegetation emit thermal radiation in the form of longwave (according to Stefan-Boltzmann law), all surfaces receive thermal radiation from each other. This represents a considerable part of the energy budget of the surfaces. The surfaces which have high view factor to other surfaces receive larger thermal radiation, as shown in Fig. 8.

\section{CONCLUSIONS}

The model system PALM 6.0 (PALM4U) is briefly introduced in this paper as a potential UCM for simulations of urban areas. The model components which essentially covers

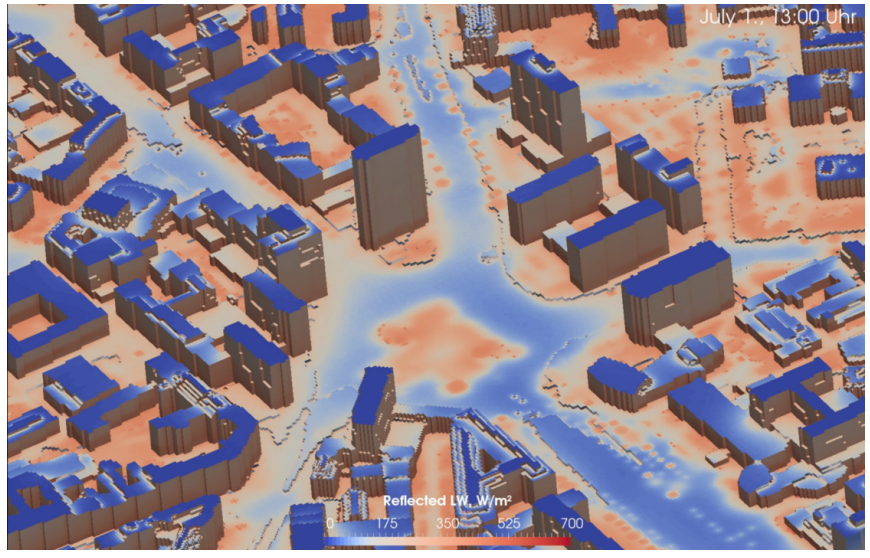

Fig. 8. Reflected longwave radiation flux received by each surface in a focus domain in the simulated area (Ernst-Reuter-Platz in Charlottenburg, Berlin) during a summer day.

all the aspects and processes in urban area are also described. We show a study case to monitor the capacity of the model and the model features. We highly recommend to use PALM4U for applications as for example urban planning, renewable energy planning, and climate change adaptation strategies in metropolitan areas.

\section{ACKNOWLEDGMENT}

This study is funded by the German Federal Ministry of Education and Research (BMBF) under grant 01LP1601 within the framework of Research for Sustainable Development (FONA; www.fona.de), which is greatly acknowledged. The German Aerospace Center (DLR) Project Management provided the input data. Simulations have been performed at the supercomputers of the North-German Supercomputing Alliance (HLRN), which also is gratefully acknowledged.

\section{REFERENCES}

[1] M.H. Salim, K.H Schlünzen, D. Grawe, M. Boettcher, A.M.U Gierisch, and B.H Fock, "The Microscale Obstacle Resolving Meteorological Model MITRAS: Model Theory" Geoscientific Model Development, vol. 11, pp. 3427-3445, 2018.

[2] M.H. Salim, K.H Schlünzen, D. Grawe, "Including trees in the numerical simulations of the wind flow in urban areas: Should we care?", Journal of Wind Engineering and Industrial Aerodynamics, vol. 144, pp. 84-95, 2015.

[3] B. Maronga, et al., "Development of a new urban climate model based on the model PALM - Project overview, planned work, and first achievements", Met. Z., pp.105-119, 2019.

[4] B. Maronga, et al., "The Parallelized Large-Eddy Simulation Model (PALM) version 4.0 for atmospheric and oceanic flows: model formulation, recent developments, and future perspectives", Geoscientific Model Development, vol. 8, pp.1539-1637, 2015.

[5] B. Maronga, et al., "Overview of the PALM model system 6.0", Geoscientific Model Development Discussions, pp.1-61, 2019.

[6] J. Resler, et al., "PALM-USM v1.0: A new urban surface model integrated into the PALM large-eddy simulation model", Geoscientific Model Development, vol. 10, pp 3635-3659, 2017 